\title{
Dyspepsia prevalence and impact on quality of life among Rwandan healthcare workers: A cross-sectional survey
}

\author{
R Bitwayiki, ${ }^{1,2}$ MBBS, MMed (IM); J T Orikiiriza, ${ }^{3,4,5} \mathrm{MB}$ ChB, MMed (Paed); F Kateera, ${ }^{3} \mathrm{MB}$ ChB, MSc; P Bihizimana, ${ }^{1,2}$ MBBS, MMed (IM); \\ B Karenzi, ${ }^{3}$ DBA, MBA, MA; P Kyamanywa, ${ }^{2}$ MB ChB, FACS; T D Walker, ${ }^{1,2}$ MBBS, FRACP, MPHTM \\ ${ }^{1}$ University Teaching Hospital, Butare, Rwanda \\ ${ }^{2}$ School of Medicine, College of Medicine and Health Sciences, University of Rwanda, Butare, Rwanda \\ ${ }^{3}$ Rwanda Military Hospital, Kigali, Rwanda \\ ${ }^{4}$ Infectious Diseases Institute, School of Medicine and Health Sciences, Makerere University, Kampala, Uganda \\ ${ }^{5}$ Department of Immunology, Trinity College, Dublin, Ireland
}

Corresponding author: T D Walker (timwalkerd@gmail.com)

\begin{abstract}
Background. Dyspepsia has been demonstrated worldwide to have major personal and societal impacts, but data on the burden of this disease in Africa are lacking.

Objective. To document the prevalence of dyspepsia and its quality-of-life impact among healthcare workers (HCWs) at Butare University Teaching Hospital (BUTH), Rwanda.

Methods. A cross-sectional survey among consenting HCWs at BUTH was conducted. Multilingual interviewers guided participants through validated questionnaires, including the Short-Form Leeds Dyspepsia Questionnaire (SF-LDQ), to detect the presence and frequency of dyspeptic symptoms, and the Short-Form Nepean Dyspepsia Index (SF-NDI), to examine the impact of dyspepsia on quality of life.

Results. The study included 378 enrolled HCWs, all of whom provided responses to the SF-LDQ and 356 of whom responded to the SF-NDI. The prevalence of dyspepsia in the study population was $38.9 \%$ (147/378). Of these 147 HCWs, 79 (53.7\%) had very mild dyspepsia, 33 (22.4\%) had mild dyspepsia, 20 (13.6\%) had moderate dyspepsia and 15 (10.2\%) had severe dyspepsia. Females were more likely to complain of dyspepsia than males (98/206 v. 49/172; odds ratio (OR) 2.3 ; $95 \%$ confidence interval (CI) 1.5 - 3.5; $p<0.001$ ). Participants with dyspepsia of at least mild severity had SF-NDI scores reflecting reduced quality of life when compared with non-dyspeptic participants (OR 17.0; 95\% CI 5.0 - 57.1; $p<0.001$ ), with most marked effects on the 'tension' and 'eating and drinking' subdomains of the SF-NDI.

Conclusion. The prevalence of dyspepsia among HCWs in Rwanda is high and is associated with lowered quality of life.

S Afr Med J 2015;105(12):1064-1069. DOI:10.7196/SAMJ.2015.v105i12.9482
\end{abstract}

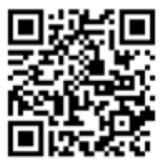

Dyspepsia is a common presenting symptom com plex in primary care all over the world, accounting for 3 - $4 \%$ of primary healthcare visits. ${ }^{[1]}$ Populationbased surveys in many countries have revealed a highly variable prevalence, from $1.8 \%$ to $57 \%$, with a global average of $20.8 \%$ in the published literature. ${ }^{[2]}$ These geographical differences are not fully accounted for by variations in symptom definition, although, as expected, large differences have been noted between less strict and more strict definitions of the dyspeptic symptom complex. ${ }^{[2]}$

Data on the frequency of dyspepsia in primary care settings in Africa are lacking, with only two prior studies having been published, both from Nigeria. ${ }^{[3,4]}$ However, while dyspepsia is a frequent reason for clinic and hospital attendance across Africa, it is not clear what proportion of symptomatic patients seek medical treatment. No previous studies have looked at the impact on quality of life of dyspeptic symptoms in Africans living in the community, although studies in other populations have shown a significant impact on quality of life, but not mortality. ${ }^{[5,6]}$

The vast majority of African studies of dyspepsia have been done in endoscopy or tertiary clinic referral populations. These populations are not representative of the community, for whom the burden of disease is unknown. This study seeks to reduce this knowledge gap by characterising the burden of dyspepsia in a presumed well, clearly defined sample of healthcare workers (HCWs) in Rwanda, to provide an accurate measure of the frequency and distribution of dyspeptic symptoms among a community-representative sample, and the quality-of-life costs associated with their symptoms.

\section{Methods}

This cross-sectional survey was conducted in Butare University Teaching Hospital (BUTH), located in the Huye District in the Southern Province of Rwanda, during October and November 2013, in conjunction with a larger study evaluating viral hepatitis burden and risk determinants among HCWs. The hospital is the sole tertiary referral centre for Southern Rwanda, with 500 beds and a catchment population of about 5 million people. The hospital also serves as a teaching site for Rwanda's sole medical school. The hospital employed 747 personnel at the time of the study: 419 clinical HCWs, 78 administrative HCWs and 250 cleaners.

\section{Study site and sample size}

The survey was powered to assess the prevalence of dyspepsia in the population. The survey's sample size was estimated at 373 subjects, based on a closed eligible population of $747 \mathrm{HCWs}$ at BUTH, with a presumed dyspepsia prevalence of $35 \%$ based on prior communitybased African studies, ${ }^{[3,4]}$ and aiming for a $5 \%$ relative standard error in the measurement of dyspepsia prevalence at an alpha level of 0.05 . All HCWs were invited to attend the study centre for free viral hepatitis testing, and then were offered participation in this study 
on their arrival, independent of the testing. Sampling of all eligible HCWs was consecutive, without random selection.

\section{Inclusion and exclusion criteria}

All consenting HCWs aged $\geq 18$ years and currently employed at BUTH were eligible for study enrolment. HCWs on leave or not currently working because of illness at the time of the study were not invited. Postgraduate students on clinical placements were considered as HCWs, but undergraduate students were not eligible.

\section{Questionnaires}

Questionnaires were administered and filled out by trained interviewers with language skills in English, French and Kinyarwanda.

The questionnaire was made up of three sections: (i) sociodemographic data; (ii) the Short-Form Leeds Dyspepsia Questionnaire (SF-LDQ); and (iii) the Short-Form Nepean Dyspepsia Index (SF-NDI).

The SF-LDQ was used to detect the presence and frequency of dyspeptic symptoms, and the SF-NDI to examine the impact of dyspepsia on quality of life.

Where possible, the survey was administered in English, but if necessary the interviewer translated the questions into French or Kinyarwanda. Prior to survey administration, an agreed wording for the oral translation of the English instruments into French and Kinyarwanda was completed, involving translation and backtranslation of the survey instrument by a group of multilingual medical experts. The LDQ and SF-NDI instruments were tested for reliability in a pilot study comprising ten HCWs eligible for study entry but not forming part of the final data set. Qualitative data were obtained from this focus group to improve the questionnaire.

\section{The Short-Form Leeds Dyspepsia Questionnaire}

The SF-LDQ consists of eight items that assess for the presence and severity of dyspepsia by measuring the frequency and severity of upper abdominal pain/discomfort, heartburn, regurgitation and nausea. It was developed from the Leeds Dyspepsia Questionnaire (LDQ), a longer form survey. ${ }^{[7]}$ Possible scores range from 0 to 32 with higher values corresponding with increasing severity of dyspepsia. The developers of the SF-LDQ have defined a score of 0 as 'no dyspepsia', a score of $1-4$ as 'very mild dyspepsia', a score of $5-8$ as 'mild dyspepsia', a score of $9-15$ as 'moderate dyspepsia' and a score $>15$ as indicative of 'severe dyspepsia. ${ }^{[8]}$ In the design of the study, we planned to analyse these results categorically but also dichotomously, with those in the 'no dyspepsia' and 'very mild dyspepsia' groups defined as non-dyspeptic (i.e. those with an SF-LDQ score of $<5$ ). The LDQ and SF-LDQ have been used in many studies and have previously been validated as accurate and reliable tools to assess the presence and severity of dyspepsia in multiple, diverse and multicultural populations. ${ }^{[9,10]}$

\section{The Short-Form Nepean Dyspepsia Index}

The SF-NDI is a questionnaire comprising ten items, making up five subscales of two items each that examine the impact of dyspepsia on various domains of the quality of life of patients, including tension/ anxiety, disruption of regular eating/drinking, knowledge and control over disease symptoms and interference with work/study ${ }^{[11]}$ It is also based on an earlier tool, the Nepean Dyspepsia Index (NDI), which was developed to study quality of life in functional dyspepsia. ${ }^{[12]}$

Each item is measured by a 5-point Likert scale ranging from 1 ('not at all' or 'not applicable') to 5 ('extremely'). Individual items in each subscale are added to obtain a score range from 10 (lowest score) to 100 (highest score). A higher score on the SF-NDI is indicative of a poorer quality of life, with patients with scores $>15$ having been shown by the designers of this instrument to have significantly reduced health-related quality of life. The NDI and SF-NDI have been used in many studies and have been validated in various populations as accurate and reliable tools to measure the impact of dyspepsia on quality of life. ${ }^{[13,14]}$

\section{Data analysis}

Key outcomes for this study include the frequency and quality-oflife impact of dyspepsia, measured by the SF-LDQ and SF-NDI, respectively. Demographic data (age, gender, marital status, department of work, profession and educational level), smoking, and alcohol, aspirin and non-steroidal drug intake are probable predictors of dyspepsia, while we expect these factors may also be co-variable with the quality-of-life impact of dyspepsia, and these were assessed in the sociodemographic section of the questionnaire.

All data were collected using a questionnaire uploaded on personal digital assistants. Query programs were written into the database to limit entry of incorrect data and to ensure data quality. The study co-ordinator and data manager reviewed the data and all associated queries daily for completeness and accuracy. Any missing data were coded as such and excluded from analysis of that variable.

The data were then transferred into SPSS (version 16.0; IBM, USA) and Stata (version 12.1; Stata Corp, USA) for data analysis. Outcome variables were the scores on SF-LDQ and SF-NDI. Comparisons of characteristics between groups of study participants were made using the $\chi^{2}$ test for categorical variables and one-way analysis of variance (ANOVA) for non-normally distributed continuous variables. Associations between predictor variables and outcomes of interest were estimated using both univariate and multivariate logistic regression, for those variables found to be significant by univariate analysis. In the final interpretation of results, a $p$-value of $<0.05$ was considered statistically significant.

\section{Ethical considerations}

Written informed consent was required from all study participants. The study was approved by the Butare University Teaching Hospital Research and Ethics Commission and the University of Rwanda School of Medicine Research Commission.

\section{Results}

The questionnaire was administered to 378 HCWs at BUTH, all of whom provided responses to SF-LDQ and 356 of whom responded to the SF-NDI.

The study population was generally young (mean 34.1 years, range $18-63)$, with a slight female preponderance (54.5\%). The majority were tertiary educated $(58.7 \%)$, but a significant proportion (18\%) had not attended secondary school. Ancillary staff, including cleaners and administration staff, outnumbered both nurses and doctors in the study population. All major hospital departments were represented, in approximate proportion to their number of staff (Table 1). The staff included in the study did not differ significantly in age, gender or department of work from those who were not included.

Using a broad definition of (any) dyspepsia, the prevalence of dyspepsia in the study population was $38.9 \%(147 / 378)$. Of these 147 HCWs, 79 (53.7\%) had very mild dyspepsia, 33 (22.4\%) mild dyspepsia, 20 (13.6\%) moderate dyspepsia and 15 (10.2\%) severe dyspepsia. Females were more likely to complain of dyspepsia than males $(98 / 206$ v. $49 / 172$, odds ratio (OR) 2.3 ; $95 \%$ confidence interval (CI) $1.5-3.5 ; p<0.001)$. Higher education levels were associated with a lower risk of dyspeptic symptoms (OR $0.6 ; p=0.015$ ), 
Table 1. Baseline characteristics and distribution of 378 study participants

\begin{tabular}{|c|c|}
\hline Variable & $n(\%)$ \\
\hline \multicolumn{2}{|l|}{ Gender } \\
\hline Male & $172(45.5)$ \\
\hline Female & $206(54.5)$ \\
\hline \multicolumn{2}{|l|}{ Age group (years) } \\
\hline $18-29$ & $125(33.1)$ \\
\hline $30-49$ & $232(61.3)$ \\
\hline$\geq 50$ & $21(5.6)$ \\
\hline \multicolumn{2}{|l|}{ Marital status } \\
\hline Single & $131(34.6)$ \\
\hline Married & $232(61.4)$ \\
\hline Divorced/separated & $4(1.1)$ \\
\hline Widowed & $9(2.4)$ \\
\hline Living together & $2(0.5)$ \\
\hline \multicolumn{2}{|l|}{ Current department of work } \\
\hline Medicine & $47(12.4)$ \\
\hline Obstetrics \& gynaecology & $30(7.9)$ \\
\hline Paediatrics & $47(12.4)$ \\
\hline Surgery & $52(13.9)$ \\
\hline Administration & $28(7.4)$ \\
\hline Laboratory & $11(2.9)$ \\
\hline Sterilisation & $6(1.7)$ \\
\hline $\begin{array}{l}\text { Other departments (cleaning } \\
\text { services, etc.) }\end{array}$ & $156(41.4)$ \\
\hline \multicolumn{2}{|l|}{ Education } \\
\hline None & $3(0.8)$ \\
\hline Primary level & $65(17.2)$ \\
\hline Secondary/vocational & $88(23.3)$ \\
\hline University/other tertiary & $222(58.7)$ \\
\hline \multicolumn{2}{|l|}{ Staffing group } \\
\hline Nurses & $123(32.5)$ \\
\hline Doctors & $40(10.6)$ \\
\hline $\begin{array}{l}\text { Others (cleaners, laboratory } \\
\text { staff, etc.) }\end{array}$ & $215(56.9)$ \\
\hline
\end{tabular}

but conventional risk factors for dyspepsia, including smoking and use of alcohol, nonsteroidal anti-inflammatory medications and aspirin, were not associated with dyspeptic symptoms in this population (Table 2). Indigestion was the most frequent symptom type reported (in 25\% of study subjects), with heartburn $(22 \%)$, regurgitation $(15 \%)$ and nausea $(9 \%)$ all both less frequently observed and showing lower frequency of lifestyle interference on the SF-LDQ (Table 3).

On multivariate analysis, gender $(p<0.001)$ and level of education $(p=0.006)$ remained highly predictive of dyspepsia,

Table 2. Dyspepsia prevalence and its association with demographic and lifestyle factors, by univariate analysis

\begin{tabular}{|c|c|c|c|}
\hline & Dyspepsia, $n /$ total (\%) & OR (CI) & $p$-value \\
\hline Overall & $147 / 378(38.9)$ & & \\
\hline \multicolumn{4}{|l|}{ Gender } \\
\hline Male & 49/172 (28.5) & (ref.) & \\
\hline Female & 98/206 (47.6) & $2.28(1.48-3.50)$ & $<0.001$ \\
\hline \multicolumn{4}{|l|}{ Marital status } \\
\hline Single & 45/131 (34.4) & & \\
\hline Married & $96 / 232(41.4)$ & & \\
\hline Widowed & $4 / 9(44.4)$ & & \\
\hline Other & $2 / 6(33.3)$ & & NS \\
\hline \multicolumn{4}{|l|}{ Age (years) } \\
\hline$<30$ & $64 / 150(42.7)$ & & \\
\hline $30-49$ & $69 / 193(35.8)$ & & \\
\hline$\geq 50$ & $14 / 35(40.0)$ & & NS \\
\hline \multicolumn{4}{|l|}{ Education level } \\
\hline Primary or secondary* & $72 / 156(46.2)$ & (ref.) & \\
\hline Tertiary & $75 / 222(33.8)$ & $0.60(0.39-0.91)$ & 0.015 \\
\hline \multicolumn{4}{|l|}{ Smoking } \\
\hline Never & $133 / 339(39.2)$ & & \\
\hline Past & $10 / 32(31.3)$ & & \\
\hline Current & $4 / 7(57.1)$ & & NS \\
\hline \multicolumn{4}{|l|}{ Alcohol } \\
\hline Nil & $101 / 241(41.9)$ & & \\
\hline Any current & $46 / 137(33.6)$ & & NS \\
\hline \multicolumn{4}{|l|}{ NSAID use } \\
\hline Never & $29 / 81(35.8)$ & & \\
\hline Past & $96 / 256(37.5)$ & & \\
\hline Current & $22 / 43(51.2)$ & & NS \\
\hline
\end{tabular}

but no other factors in the model were statistically significant, with the exception of the consumption of alcohol, which was associated with a borderline lower risk of dyspepsia $(p=0.043)$.

Participants with dyspepsia had reduced quality-of-life scores, as measured by the SF-NDI, when compared with nondyspeptic participants $(p<0.001)$, the most marked effects being on the tension and eating and drinking subdomains of the SF-NDI. An appreciable difference in quality of life was apparent, with the most marked reduction seen in subjects with at least mild severity dyspepsia, compared with those suffering no or very mild dyspepsia (sum of squares by ANOVA 779.27; $p<0.001$ ) (Table 4).

As an alternative measure, the proportion of subjects with reduced quality of life (def- ined as an SF-NDI of $\geq 15$ points, consisting of the bottom $8 \%$ of the population) in each dyspeptic symptom group was also calculated, with $1.4 \%$ and $10.7 \%$ of non- and very mildly dyspeptic patients meeting this threshold, compared with $25.8 \%, 31.6 \%$ and $33.3 \%$ of mildly, moderately and severely dyspeptic patients.

Analysis of the predominant symptom and covariance in symptoms on the SF-LDQ was also undertaken, with the goal of defining patients into separate dyspeptic symptom complex groups. These were defined as predominant epigastric pain ('ulcer-like'), predominant heartburn or regurgitation ('reflux-like'), predominant nausea ('dysmotility-like') and mixed groups. Of 90 patients with dyspepsia and a predominant symptom available for analysis, $38(42.2 \%)$ described ulcer-like symptoms, 37 (41.1\%) 
reflux-like symptoms, 8 (8.9\%) dysmotility-like symptoms, and 7 (7.8\%) mixed symptoms.

There was a significantly higher SF-LDQ $(t=-3.314, p=0.009)$ among patients with reflux-like or mixed symptoms (mean 8.25) than among those with ulcer-like or dysmotility-like symptoms (mean 4.94). However, quality of life measured by the SF-NDI was not significantly different between these groups (means $13.16 \mathrm{v}$. $12.48 ; p>0.05)$.

Table 3. Frequency and lifestyle interference of each SF-LDQ symptom type in study subjects

\begin{tabular}{|c|c|c|}
\hline Symptom type & $\begin{array}{l}\text { Symptom } \\
\text { frequency (\%) }\end{array}$ & $\begin{array}{l}\text { Interference with } \\
\text { lifestyle frequency (\%) }\end{array}$ \\
\hline \multicolumn{3}{|l|}{ Indigestion } \\
\hline Never & $281(74)$ & $322(85)$ \\
\hline$<$ monthly & $34(9)$ & $10(3)$ \\
\hline$>$ monthly, < weekly & $31(8)$ & $20(5)$ \\
\hline$>$ weekly, < daily & $23(6)$ & $17(5)$ \\
\hline$>$ daily & $9(2)$ & $9(2)$ \\
\hline \multicolumn{3}{|l|}{ Heartburn } \\
\hline Never & $298(79)$ & $329(87)$ \\
\hline$<$ monthly & $26(7)$ & $11(3)$ \\
\hline$>$ monthly, < weekly & $26(7)$ & $14(4)$ \\
\hline$>$ weekly, < daily & $18(5)$ & $14(4)$ \\
\hline$>$ daily & $10(3)$ & $10(3)$ \\
\hline \multicolumn{3}{|l|}{ Regurgitation } \\
\hline Never & $320(85)$ & $353(93)$ \\
\hline$<$ monthly & $23(6)$ & $7(2)$ \\
\hline > monthly, < weekly & $18(5)$ & $8(2)$ \\
\hline$>$ weekly, < daily & $12(3)$ & $6(2)$ \\
\hline$>$ daily & $5(1)$ & $4(1)$ \\
\hline \multicolumn{3}{|l|}{ Nausea } \\
\hline Never & $343(91)$ & $355(94)$ \\
\hline$<$ monthly & $20(5)$ & $9(2)$ \\
\hline$>$ monthly, < weekly & $4(1)$ & $4(1)$ \\
\hline$>$ weekly, < daily & $6(2)$ & $5(1)$ \\
\hline$>$ daily & $5(1)$ & $5(1)$ \\
\hline
\end{tabular}

\section{Discussion}

Dyspepsia affected more than a third of Rwandan HCWs in this study, and had a significant impact on wellbeing, with symptoms severe enough to impair the quality of life of almost a third of affected HCWs. Ulcer-like and reflux-like symptoms were equally common, perhaps surprisingly, given how infrequently gastro-oesophageal reflux is diagnosed in African endoscopic series. The observed dyspepsia prevalence is at the high end of the range garnered from studies in other parts of the world, although these frequencies depend greatly on the definition of dyspepsia used in the study. ${ }^{[2]}$

One of the key challenges in studying dyspepsia is a lack of a clear, agreed standard definition of dyspeptic symptoms. Older studies tended to use broad descriptive questions on the presence of epigastric discomfort. More recently, the Rome II-III criteria have been developed, which attempt to draw a distinction between reflux symptoms and dyspepsia in order to distinguish gastro-oesophageal reflux disease from functional dyspepsia, but have met with a mixed reception. As a result, a variety of definitions remain in use, with simplicity, prior multicultural validity and ease of administration being our key criteria in choosing the SF-LDQ and SF-NDI for this study.

The SF-LDQ is based upon a broad definition of dyspepsia, not attempting to exclude reflux symptoms from the definition, and assessing symptoms over a 2-month period. In a recent meta-analysis by Ford et al. ${ }^{[2]}$ using a broad definition of this type gave a $29.5 \%$ pooled prevalence of dyspepsia. However, very few of the studies previously reported have used face-to-face interviews; those that did reported higher than average prevalences, pointing to a possible systematic effect of this data acquisition method on reported dyspepsia prevalence. While the SF-LDQ only assesses a limited range of dyspeptic symptoms, and does not include assessment of postprandial symptoms, which have been highly prevalent in Western studies, correlation between the SF-LDQ and other lengthier tools that include such symptoms has been high in published validation studies. ${ }^{[8,9]}$

As a symptom complex, uninvestigated dyspepsia does not have a single underlying pathological mechanism. Putative contributing factors include Helicobacter pylori infection, gastric acid and gastric dysmotility. ${ }^{[15]}$ In Western populations, about $80 \%$ of patients with uninvestigated dyspepsia have a final diagnosis of functional dyspepsia following investigation. ${ }^{[6]}$ The pathogenesis of functional dyspepsia is debated, and has not been well studied in areas with a high prevalence of $H$. pylori infection, although a recent observational study suggested a role for $H$. pylori and associated microscopic duodenitis. ${ }^{[16]}$

Traditional risk factors such as alcohol, smoking and nonsteroidal anti-inflammatory drug (NSAID) use were not found

Table 4. Severity of dyspepsia and impact on quality of life, as measured by mean SF-NDI scores in 356 subjects ${ }^{*}$

\begin{tabular}{|c|c|c|c|c|c|c|}
\hline & No dyspepsia & Very mild dyspepsia & Mild dyspepsia & Moderate dyspepsia & Severe dyspepsia & $p$-value \\
\hline SF-NDI total score & 10.31 & 11.21 & 13.90 & 14.47 & 15.20 & $<0.001$ \\
\hline SF-NDI: Tension & 2.10 & 2.57 & 3.39 & 3.68 & 3.93 & $<0.001$ \\
\hline $\begin{array}{l}\text { SF-NDI: Interference with } \\
\text { ADLs }\end{array}$ & 2.06 & 2.24 & 2.68 & 2.84 & 2.87 & $<0.001$ \\
\hline SF-NDI: Eating/drinking & 2.04 & 2.19 & 2.71 & 3.37 & 3.07 & $<0.001$ \\
\hline $\begin{array}{l}\text { SF-NDI: Knowledge/ } \\
\text { control }\end{array}$ & 2.06 & 2.11 & 2.55 & 2.16 & 2.67 & 0.004 \\
\hline SF-NDI: Work/study & 2.05 & 2.11 & 2.58 & 2.42 & 2.67 & $<0.001$ \\
\hline
\end{tabular}


to be associated with symptoms of dyspepsia in our study. While this initially appears surprising and is in contrast to the wider literature, ${ }^{[2]}$ there are several good reasons why this might be the case among HCWs. First, HCWs are more likely to have an understanding of the link between such risk factors and the onset of dyspeptic symptoms. Secondly and more speculatively, HCWs might be more amenable to changing their behaviours in response to this knowledge, even without medical attention. As this study was cross-sectional, it was unable to examine the effects of dyspepsia on these health-related behaviours over time.

Among Rwandan HCWs in this study, women were more likely to complain of dyspeptic symptoms than men, and more likely to suffer lower quality of life as a result. This is in contrast with the only previous studies of dyspepsia in Africa, conducted in Nigeria, ${ }^{[3,4]}$ where men had a similar rate to women (OR 0.93; 95\% CI $0.73-1.19 .{ }^{[2]}$ It should be noted that there are significant cultural variations between African nations, and it may be that the role of gender in illness behaviour is significantly different in Nigeria and Rwanda, as has been noted in other illnesses in disparate African contexts. The findings in our study are, however, quite consistent with the broader population-based international literature, where in a recent meta-analysis by Ford et al. ${ }^{[2]}$ the pooled OR for women with dyspepsia requiring investigation was 1.24.

Higher levels of education were protective in this study of HCWs, although this may not be generalisable to non-healthcare education, as most participants in this study had higher qualifications in the health sciences. Previous studies in other populations have not shown any such association, ${ }^{[6]}$ and it is possible that this represents a unique finding, as a result of the characteristics of this population. While $H$. pylori positivity rates were not measured in this study, it is interesting to note that higher education levels have been associated with lower rates of this infection among HCWs in other parts of the world, and this could explain this result. ${ }^{[17]}$

The presence and relative frequency of two major dyspeptic symptom complexes in this study is revealing. While the previous literature describes most African patients as suffering from ulcer-like symptoms, in our study reflux-like symptoms were just as common, and just as likely to be rated as the major symptom. While the SF-LDQ has two of four question groups related to reflux symptoms (heartburn and regurgitation) and this may explain the higher SF-LDQ scores among subjects with a predominantly reflux-based symptom complex, the quality-of-life impact measured by SF-NDI was similar for both groups, as with studies in other populations. ${ }^{[1,14]}$

In these previous studies, ulcer-like and reflux-like symptom complexes were associated with moderate responses to acid suppression with omeprazole, in contrast to those with dysmotilitylike symptoms. ${ }^{[18]} H$. pylori eradication has also been only modestly effective in treating dyspeptic symptoms in low-prevalence populations in the Western world. Whether these data can be extrapolated to Africa, where $H$. pylori carriage rates are much higher and the mind-body interplay around dyspepsia may differ, is unclear.

\section{Study limitations and strengths}

We studied HCWs, a relatively well-educated population with greater than average access to healthcare knowledge and resources. As such, especially given the protective effect of higher levels of education apparent in this population, the study is likely to underestimate rates of dyspepsia in the general Rwandan population. Other potential reasons for a lower rate of dyspepsia in this population could include better quality of life, as lower quality of life has been shown to increase the incidence of new dyspepsia in a previous study. ${ }^{[19]}$ The study population was based in a provincial town, and results may not be easily extrapolated to people leading a traditional village lifestyle.

However, the study being performed in HCWs also gives it some notable strengths. Firstly and most importantly, HCWs have a shared linguistic and pathophysiological understanding of clinical symptoms, which aids the validity of comparison with international cohorts by reducing the cultural gap. Secondly, most study subjects were able to understand both English and French, reducing the need to translate the tools. Thirdly, a relatively small, geographically close study population aided study logistics and maximised recruitment.

Our study nonetheless has several limitations. The cross-sectional methodology used made it impossible to determine the direction of effect in the association between dyspepsia and lower quality of life. It is possible that dyspepsia causes lower quality of life, but equally plausible that patients with lower quality of life might be more susceptible to the onset of dyspeptic symptoms; this complex interplay needs further study. Definitions of dyspepsia are culturally and linguistically bounded, and despite our efforts to translate and validate the SF-LDQ and SF-NDI, it is impossible to know whether these definitions fill exactly the same linguistic and cultural space when translated. Our study was of uninvestigated dyspepsia, and thus the study methodology was unable to distinguish organic and functional causes of dyspepsia, which might have a different natural history in African populations, although the quality-of-life impact of each has been similar in other populations. ${ }^{[6]}$ This should be the subject of future research.

\section{Conclusion}

Dyspepsia is a significant symptom complex among HCWs in this Rwandan tertiary institution, and has a major quality-of-life impact in a proportion of these. Reflux-like symptoms were just as common as ulcer-like symptoms, in contrast to findings in endoscopy-based studies. HCWs have high levels of uncontrolled symptoms, and rates may be even higher in the general community because of less access to healthcare resources.

More resources need to be devoted to the diagnosis and management of upper abdominal symptoms in Rwanda and other similar resourcepoor areas. Finally, further research in population-based cohorts is needed to characterise prevalence, symptom-complex patterns and underlying diagnoses in the wider African population.

Author contributions. TDW conceived the study; RB, BK, PK and TDW designed the study; $\mathrm{PB}, \mathrm{RB}$ and JTO carried out and supervised the interviews; RB, FK and TDW interpreted the data; TDW and RB drafted the manuscript; and RB, PB, FK, JTO, PK, BK and TDW critically revised the manuscript for intellectual content. All the authors read and approved the final manuscript. TDW and RB are guarantors of the paper.

Acknowledgements. We thank Profs Nick Talley and Brendan Delaney for kind permission to use the SF-NDI and SF-LDQ tools, and Rwanda Military Hospital, as well as all staff at Butare University Teaching Hospital for their valued support of this study.

Funding. Rwanda Military Hospital provided study staff, equipment and facilitation for the conduct of this study. The drafting of this study was supported in part by Africa Research Initiatives and Support Enterprise (ARISE), Grant Number W.07.10.102 funded by The Netherlands African Partnership for Clinical Trials and Capacity Building Program (NACCAP) of The Netherlands Organisation for Scientific Research The Netherlands Foundation for the Advancement of Tropical Research (NWO/WOTRO). Its contents are solely the responsibility of the authors and do not necessarily represent the official views of the supporting offices. 


\section{References}

1. Bodger K, Eastwood PG, Manning SI, et al. Dyspepsia workload in urban general practice and implications of the British Society of Gastroenterology Dyspepsia Guidelines (1996). Aliment Pharmacol Ther 2000;14(4):413-420.

2. Ford AC, Marwaha A, Sood R, et al. Global prevalence of, and risk factors for, uninvestigated dyspepsia: A meta-analysis. Gut 2015;64:1049-1057. [http://dx.doi.org/10.1136/gutinl-2014-307843]

3. Thezue $\mathrm{CH}$, Oluwole FS, Onuminya JE, et al. Dyspepsias among the highlanders of Nigeria: An epidemiological survey. Afr J Med Med Sci 1996;25(1):23-29.

4. Holcombe C, Omotara BA, Padonu MK, et al. The prevalence of symptoms of dyspepsia in north eastern Nigeria: A random community based survey. Trop Geogr Med 1991;43(1-2):209-214.

5. Ford AC, Forman D, Bailey AG, et al. Effect of dyspepsia on survival: A longitudinal 10-year follow-up Ford AC, Forman D, Bailey AG, et al. Effect of dyspepsia on survival: A longitudinal 10-yer
study. Am J Gastroenterol 2012;107(6):912-921. [http://dx.doi.org/10.1038/ajg.2012.69]

6. Aro P, Talley NJ, Agreus L, et al. Functional dyspepsia impairs quality of life in the adult population. Aliment Pharmacol Ther 2011;33(11):1215-1224. [http://dx.doi.org/10.1111/j.13652036.2011.04640.x]

7. Moayyedi P, Duffett S, Braunholtz D, et al. The Leeds Dyspepsia Questionnaire: A valid tool for measuring the presence and severity of dyspepsia. Aliment Pharmacol Ther 1998;12(12):1257-1262. 8. Fraser A, Delaney BC, Ford AC, et al. The Short-Form Leeds Dyspepsia Questionnaire validation study. Aliment Pharmacol Ther 2007;25(4):477-486. [http://dx.doi.org/10.1111/j.1365-2036.2006.03233.x]

9. Gatta L, Moayyedi P, Tosetti C, et al. A validation study of the Italian Short-Form Leeds Dyspepsia Questionnaire. Intern Emerg Med 2010;5(6):501-506. [http://dx.doi.org/10.1007/s11739-010-0467-5']

10. Mahadeva S, Chan WK, Mohazmi M, et al. Validation study of the Leeds Dyspepsia Questionnaire in a multi-ethnic Asian population. J Gastroenterol Hepatol 2011;26(11):1669-1676. [http://dx.doi. org/10.1111/j.1440-1746.2011.06806.x]

11. Talley NJ, Verlinden M, Jones M. Quality of life in functional dyspepsia: Responsiveness of the Nepean Dyspepsia Index and development of a new 10-item short form. Aliment Pharmacol Ther 2001;15(2):207-216.
12. Talley NJ, Verlinden $M$, Jones $M$. Validity of a new quality of life scale for functional dyspepsia: A United States multicenter trial of the Nepean Dyspepsia Index. Am J Gastroenterol 1999;94(9):23902397. [http://dx.doi.org/10.1111/j.1572-0241.1999.01363.x]

13. Tian XP, Li Y, Liang FR, et al. Translation and validation of the Nepean Dyspepsia Index for functional dyspepsia in China. World J Gastroenterol 2009;15(25):3173-3177.

14. Mahadeva S, Wee HL, Goh KL, et al. Quality of life in South East Asian patients who consult for dyspepsia: Validation of the short form Nepean Dyspepsia Index. Health Qual Life Outcomes 2009; $7: 45$. [http://dx.doi.org/10.1186/1477-7525-7-45]

15. Bytzer P, Talley NJ. Dyspepsia. Ann Intern Med 2001;134(9):815-822. [http://dx.doi.org/10.7326/00034819-134-9_Part_2-200105011-00004]

16. Mirbagheri SS, Mirbagheri SA, Nabavizadeh B, et al. Impact of microscopic duodenitis on symptomatic response to Helicobacter pylori eradication in functional dyspepsia. Dig Dis Sci 2015;60(1):163-167. [http://dx.doi.org/10.1007/s10620-014-3285-1]

17. Triantafillidis JK, Gikas A, Hyphantis T, et al. Helicobacter pylori infection in hospital workers over a 5-year period: Correlation with demographic and clinical parameters. J Gastroenterol 2002;37(12):1005-1013.

18. Talley NJ, Meineche-Schmidt V, Pare P, et al. Efficacy of omeprazole in functional dyspepsia: Doubleblind, randomized, placebo-controlled trials (the Bond and Opera studies). Aliment Pharmacol Ther 1998;12(11):1055-1065.

19. Ford AC, Forman D, Bailey AG, et al. Initial poor quality of life and new onset of dyspepsia: Results from a longitudinal 10-year follow-up study. Gut 2007;56(3):321-327. [http://dx.doi.org/10.1136/ gut.2006.099846]

Accepted 5 October 2015. 